\title{
Colorectal Cancer in Inflammatory Bowel Disease
}

\author{
Jonathan Potack and Steven H. Itzkowitz \\ Division of Gastroenterology, Department of Medicine, Mount Sinai School of Medicine, New York City, United States
}

Patients with long-standing inflammatory bowel disease have an increased risk of developing colorectal cancer (CRC). CRC risk increases with longer duration of colitis, greater anatomic extent of colitis, the presence of primary sclerosing cholangitis, family history of CRC and severity of inflammation of the colon. Chemoprevention includes aminosalicylates, ursodeoxycholic acid, and possibly folic acid. To reduce CRC mortality in IBD, colonoscopic surveillance remains the major way to detect early mucosal dysplasia. When dysplasia is confirmed, proctocolectomy is considered for these patients. Ulcerative colitis patients with total proctocolectomy and ileal pouch anal-anastomosis have a rather low risk of dysplasia in the ileal pouch, but the anal transition zone should be monitored periodically. New endoscopic and molecular screening approaches may further refine our current surveillance guidelines and our understanding of the natural history of dysplasia. (Gut and Liver 2008;2:61-73)

Key Words: Inflammatory bowel disease; Dysplasia; Colorectal neoplasms

\section{INTRODUCTION}

The two forms of inflammatory bowel disease (IBD), Crohn's disease (CD) and ulcerative colitis (UC), are characterized by chronic, relapsing inflammation of the intestines. First described in a report by Crohn and Rosenberg in 1925, ${ }^{1}$ colorectal cancer (CRC) in patients with IBD has long been recognized. Even years after their disease is controlled with medications, IBD patients still carry the risk of developing cancer. Together with the hereditary syndromes of familial adenomatous polyposis and hereditary nonpolyposis colorectal cancer, IBD is among the top three high-risk conditions for CRC. Although cancer in other organs can occur in patients with IBD, CRC is the most common site of cancer. This review will focus mainly on the problem of CRC in patients with IBD.

\section{PREVALENCE AND INCIDENCE OF CRC}

The risk of CRC in IBD has been difficult to estimate in the past due, in large part, to methodological issues. Early estimates from tertiary referral centers often included patients with disproportionately severe disease, and sometimes included patients who presented already with their cancer, thereby likely overestimating the risk. For example, the probability of developing CRC was as high as $60 \%$ after 40 years of UC. ${ }^{2}$ Later population-based studies tended to include more patients with limited disease and reported significantly lower rates of CRC. ${ }^{3}$ A meta-analysis in 2001 by Eaden and colleagues attempted to clarify some of these differences. It included 116 studies from a wide array of centers and geographic sites. The prevalence of CRC in UC was $3.7 \%$. However, the incidence, which measures risk over time, was $0.3 \% /$ year or 3 cases of CRC per 1,000 patient-years of follow-up. ${ }^{4}$ Based on long-term follow-up in a subset of studies included in the meta-analysis, Eaden's group estimated the cumulative risk of CRC in UC to be $1.6 \%$ at 10 years, $8.3 \%$ at 20 years and $18.4 \%$ at 30 years.

Since the publication of that meta-analysis, several population-based studies have reported lower risks of CRC. Palli et al reported an annual incidence of $0.13 \%$ in a cohort of 689 UC patients diagnosed between 1978 and $1992 .^{5}$ Bernstein et al reported an annual incidence of $0.2 \%$ in a cohort of 2672 UC patients diagnosed be-

Correspondence to: Steven H. Itzkowitz

Division of Gastroenterology, Department of Medicine, Box 1069, Mount Sinai School of Medicine, One Gustave Levy Place, New York, NY 10029, United States

Tel: +212-659-9697, Fax: +212-849-2574, E-mail: steven.itzkowitz@mountsinai.org Received on August 25, 2008. Accepted on August 27, 2008. 
tween 1984 and 1997. ${ }^{6}$ A third study from Hungary followed 723 patients diagnosed with UC over 30 years and found an annual incidence of colorectal cancer of $0.15 \%{ }^{7}$

Just as the absolute risk of colorectal cancer seems to be decreasing, so too, is the relative risk of CRC in IBD patients compared to the general population. In fact, recent population-based studies do not show a statistically significant increased in risk of CRC in IBD compared to control populations. One way to calculate relative risk is with a standardized morbidity ratio (SMR) which is the observed cancers in a UC cohort divided by the number of expected cancers derived from a non-IBD population. ${ }^{8}$ Some studies have shown a 2.5 to 6-fold increase in CRC as compared to controls. ${ }^{6,9,10}$ However, newer studies have not confirmed this. Three recent population-based studies have calculated the SMR for CRC to be between 1.05 and 1.79; values that reflected statistically non-significant increases over non-IBD populations. ${ }^{5,11,12}$

The incidence of dysplasia in UC has been more difficult to measure than cancer given that it is a more subjective finding. Furthermore, selection bias is introduced since dysplasia can only be found in patients undergoing colonoscopic surveillance. A Swedish study with 3664 patient-years of follow-up found that dysplasia occurred in $24 \%$ of patients during the study, with an incidence rate for developing dysplasia of $2.4 \% /$ year. $^{13}$ Two additional long-term studies showed a cumulative risk of dysplasia of $9.2 \%$ at 25 years $^{14}$ and $7.7 \%$ at 20 years and $15.8 \%$ at 30 years. ${ }^{15}$

Crohn's disease involving the colon (as opposed to pure ileitis) carries a similar risk of CRC to that of UC given comparable disease extent. Even without controlling for these factors, a large population based study from Manitoba, Canada demonstrated CRC risk in Crohn's disease (RR 2.64; 95\% CI 1.69-4.12) equal to that in UC patients (RR 2.75; 95\% CI 1.91-3.97). ${ }^{16}$ Another study compared 28 patients with CRC in Crohn's disease to 52 patients with CRC in UC and found similar age at cancer diagnosis, duration of IBD, multiplicity, distribution of cancers and overall 5-year survival rates. ${ }^{17}$ While future studies will help to further define both cancer and dysplasia risks, in the meantime it is best to still consider IBD patients at high-risk for developing CRC and monitor them accordingly.

\section{CLINICAL FEATURES OF COLITIS-ASSOCIATED CRC}

Compared with sporadic colorectal carcinoma (SCC), CRC arising in patients with IBD has several distinguishing clinical features. Colitis-associated colorectal cancer (CAC) affects individuals at a younger age than the general population. The mean age of CRC diagnosis in the Eaden meta-analysis was 43.2 years. ${ }^{4}$ CAC progresses to invasive adenocarcinoma from flat and nonpolypoid dysplasia more frequently than SCC. CACs more often have a higher proportion of mucinous and signet ring cell histology, and in some studies, have a more proximal colonic distribution. There is a higher rate of synchronous lesions in IBD patients. The multifocality of CAC is likely due to the broad field-effect of mucosal inflammation contributing to the development of neoplasia.

\section{SPORADIC COLON CANCER}

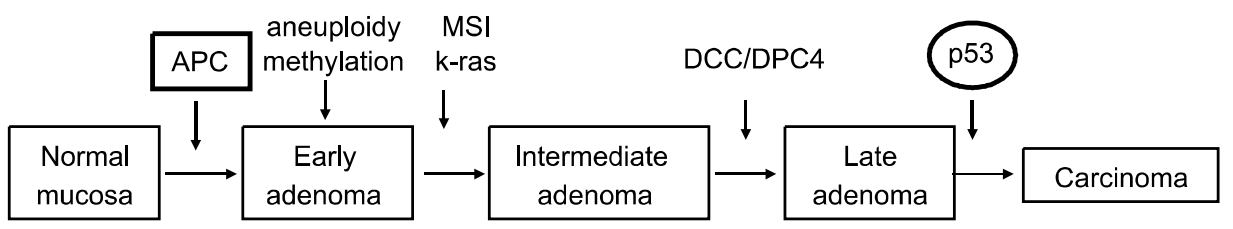

\section{COLITIS-ASSOCIATED COLON CANCER}

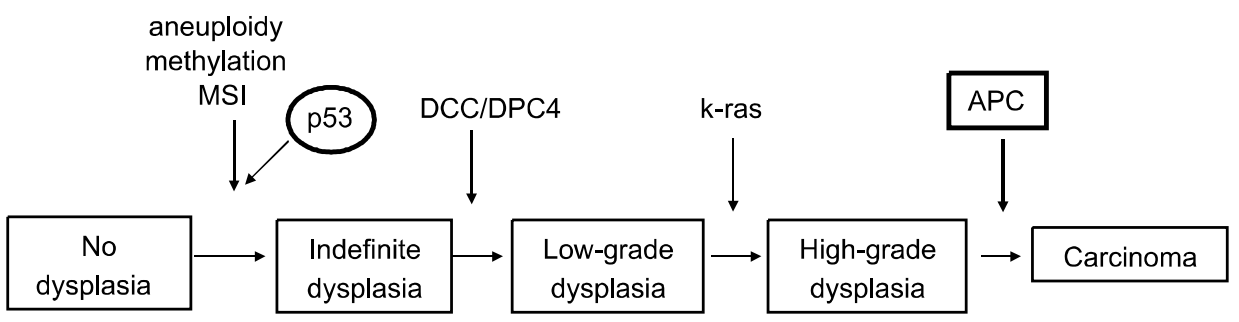

Fig. 1. Comparison of molecular alterations in sporadic colon cancer and colitis-associated colon cancer.

MSI, microsatellite instability; DCC/DPC4, deleted in colon cancer/deletedin pancreatic cancer-4; APC, adenomatous polyposis coli; k-ras, Kirsten-ras.

Modified from Ref. 61, with permission. 
Finally, the sequence of molecular events leading from dysplasia to invasive adenocarcinoma in CAC is different from SCC. For example, APC loss of function, considered to be a very common early event in SCC, is much less frequent and usually occurs late in the colitis-associated dysplasia-carcinoma sequence. Conversely, p53 mutations in sporadic neoplasia usually occur late in the adenoma-carcinoma sequence, whereas in patients with colitis, p53 mutations occur early and are often detected in mucosa that is non-dysplastic or indefinite for dysplasia (Fig. 1). These differences imply that to manage CRC risk in IBD patients, one must understand the special risk factors and biology of dysplasia in these chronic inflammatory conditions.

\section{CRC RISK FACTORS}

Several factors have been identified which either increase or decrease CRC risk in the setting of IBD (Table 1). Duration of colitis is one of the most important factors that increase the risk of CRC. For reasons that are unclear, CRC rarely develops before 7-8 years of colitis. Of course, it is often difficult to know the precise date of onset of disease since many patients may have symptoms for a long time without seeking medical attention. Nonetheless, after the first decade of disease, the risk begins to rise. In one recent referral-based study, the cumulative incidence of CRC was $2.5 \%, 7.6 \%$, and $10.8 \%$ after follow-up periods of 20,30 , and 40 years, respectively. ${ }^{15}$

Extent of colitis is another important risk factor for the development of CRC; the more colonic surface that is involved with colitis, the greater the risk. How disease extent is measured differs between studies. For example, an early, yet classic population-based study of over 3,000 UC patients in Sweden used barium enema

Table 1. Factors Associated with Colorectal Cancer (CRC) Risk in Inflammatory Bowel Disease (IBD)

Factors that increase CRC risk

- Longer duration of colitis

- Greater extent of colonic involvement

- Family history of colorectal cancer

- Primary sclerosing cholangitis

- Severity of histologic inflammation

- Young age of IBD onset (some studies)

- History of dysplasia

Factors that decrease CRC risk

- Prophylactic total proctocolectomy

- Surveillance colonoscopy

- Chemoprevention findings to demonstrate that patients with proctitis had a standardized incidence ratio (SIR) of 1.7 [95\% confidence interval (CI) 0.8-3.2] compared with age-matched population controls without UC, whereas those with left-sided and universal colitis had SIRs of 2.8 (95\% CI 1.6-4.4), and 14.8 (95\% CI 11.4 to 18.9 ), respectively. ${ }^{18}$ There is now general agreement that there is little or no increased risk of CRC in patients with proctitis or proctosigmoiditis, whereas the risk is intermediate in those with left-sided colitis and highest with pan-colitis. ${ }^{18,19}$ Backwash ileitis, theoretically representing the greatest extent of disease because of ileal involvement, was reported to carry a high risk of $\mathrm{CRC},{ }^{20}$ but this has not been confirmed by others. ${ }^{21}$

Because inflammation is the presumed substrate from which cancers arise in IBD, it seems obvious that studies would have examined the relationship between severity of inflammation and CRC risk. Ironically, it was not until recently that this concept was formally substantiated. Documenting active inflammation is best done by endoscopic and histologic means, rather than by clinical symptoms. A case-control study from St. Mark's Hospital, London, demonstrated that greater degrees of active inflammation, by colonoscopy (odds ratio 2.5) or histology (OR 5.1) were associated with greater rates of $\mathrm{CRC}^{22}$ Using a retrospective cohort study design, data from a surveillance cohort at The Mount Sinai Hospital, New York, showed that histologic inflammation severity analyzed over time was significantly correlated with subsequent development of both high-grade dysplasia and CRC. ${ }^{23}$ Furthermore, a study of 30 colectomy specimens showed that dysplasia and CRC can arise in areas of microscopic colitis that are proximal to areas of gross colitis, suggesting that indeed histologic changes, even without macroscopic alterations visible on colonoscopy, might better define disease extent for the purposes of cancer risk. ${ }^{24}$

IBD patients with a family history of CRC have at increased risk of CRC. An odds ratio of 2-3 for the development of CRC in patients with a family history of CRC has been described in both case control and population-based studies. ${ }^{8}$

Both UC and Crohn's disease patients with primary sclerosing cholangitis (PSC) are at significantly increased risk for developing colorectal neoplasia. In a population-based Swedish study, the cumulative incidence of CRC in UC patients with PSC was $33 \%$ at 20 years. ${ }^{25}$ Others reported an adjusted relative risk (RR) for dysplasia or cancer of 3.15 (95\% CI, 1.37 to 7.27 ) in patients with PSC and UC as compared to patients with only UC. ${ }^{26}$ The mechanism by which PSC increases 
the risk of neoplasia is unclear. Some evidence suggests that patients with PSC have a longstanding quiescent colitis and the prolonged duration of colitis contributes to the increased risk. ${ }^{27}$ Others have pointed to the apparent chemoprotective effects of ursodeoxycholic acid on reducing CRC risk in PSC patients as evidence that PSC itself directly increases the risk of neoplasia. ${ }^{28}$ Given the high risk of neoplasia, current practice guidelines recommend all patients with PSC not previously known to have IBD should undergo a colonoscopy to determine their status. For those patients with IBD, screening and subsequent surveillance should begin at the diagnosis of PSC.

Young age at onset of colitis has been reported to be an independent risk factor for CRC in some studies, but controversy exists as to whether this effect is independent of total disease duration. Indeed, the interval between onset of colitis and development of CRC may be the same in young and older patients. ${ }^{11}$

\section{DIAGNOSING DYSPLASIA}

\section{Macroscopic classification of dysplasia}

Dysplasia in IBD is often classified macroscopically as raised or flat, depending on whether it corresponds to an endoscopically visible lesion. Morphologically raised lesions can appear as polypoid or as non-discrete plaques or patches. Such lesions can blend easily with the gross inflammatory abnormalities commonly encountered in colons with IBD, making their endoscopic detection more difficult. Raised lesions are occasionally called DALMs (dysplasia associated lesion or mass), and some observers use the term DALM interchangeably with raised lesions. However, if a raised lesion can be completely resected endoscopically, studies suggest that such patients can be managed conservatively without the need for colectomy (see below). It is the raised lesion that cannot be endoscopically resected in its entirety that is of greater concern, and is more appropriately referred to as a DALM.

The reputation of DALMs as ominous harbingers of malignancy dates back over two decades to the study of Blackstone and colleagues who discovered cancers at colectomy in 7 of 12 DALM-bearing colons, including 5 with preoperative biopsies showing only mild or moderate dysplasia (currently called low-grade dysplasia). ${ }^{29}$ Subsequent reports from ten surveillance programs also described cancers in 17 of $40(43 \%)$ colectomies performed because of a DALM. ${ }^{30}$ Thus, it is generally recommended that the presence of a DALM warrants a colectomy irrespective of the grade of dysplasia in pre- operative biopsies. This concept is analogous to the management of a sporadic adenoma that cannot be completely resected endoscopically, although the type of surgery might differ.

More recently, experience has taught that not all types of visible dysplasia in patients with IBD carry the same significance. For one thing, a patient with colitis could conceivably develop an adenomatous polyp unrelated to colitis: for example, a sporadic adenoma located in the proximal colon of a patient with left-sided ulcerative colitis. In this situation, although the adenoma can usually be managed with conventional endoscopic polypectomy, it is advisable to also biopsy the mucosa surrounding the polyp base to make sure it does not reveal underlying colitis or dysplasia. Even in areas of active colitis, one can sometimes encounter "adenomalike" polyps. ${ }^{31,32}$ These polyps can look endoscopically indistinguishable from sporadic adenomatous polyps, and even histologically, it may be difficult to distinguish a dysplastic polyp in colitis from a true sporadic adenoma. Nevertheless, two important studies demonstrated that these "adenoma-like polyps" in areas of colitis can safely be managed with endoscopic removal without the need for urgent colectomy. A 1999 study from The Mount Sinai Hospital in New York reported that endoscopic polypectomy in a cohort of 48 UC patients with a total of 70 adenoma-like dysplastic polyps (including 3 with high-grade dysplasia), resulted in no adverse outcomes during a mean follow-up period of $4.1 \mathrm{y}{ }^{31} \mathrm{~A}$ concurrent study from Brigham and Women's Hospital reached a similar conclusion ${ }^{32}$ that was confirmed upon longer follow-up. ${ }^{33}$ There are no standardized criteria for what qualifies as an "adenoma-like polyp", individual endoscopists need to make the decision on a case by case basis. In the future, molecular markers may ultimately afford a more objective means of making these distinctions. For the time being, current management recommendations suggest that if a dysplastic polyp in the setting of colitis has been endoscopically removed in its entirety, the base of the polyp has been separately biopsied, and there is no dysplasia in the polyp base or elsewhere in the colon on segmental biopsies, the patient can be followed expectantly (see below).

More problematic is the issue of flat dysplasia in colitis. Whereas sporadic cancers commonly arise from polypoid lesions, IBD-associated cancers often emerge from flat, or even non-visible, dysplastic lesions. In the context of colitis, the term "flat dysplasia" has been used by endoscopists to describe a lesion that was not grossly visible by conventional white light endoscopy, but was only detected microscopically in random biopsies from 
unremarkable mucosa. Accurate detection therefore depended on adequate mucosal sampling by the endoscopist. This led to estimates indicating that in order to exclude dysplasia with $90 \%$ certainty, ${ }^{33}$ random biopsy specimens were required throughout the colon, and to increase the accuracy to $95 \%$, nearly twice that number were required. ${ }^{34}$ With recent advances in endoscopic technology (such as chromoendoscopy and high-definition endoscopes, see below) it seems that truly invisible dysplasia is less common than originally thought. Nonetheless, current surveillance strategies still recommend taking four- quadrant biopsy specimens every $10 \mathrm{~cm}$ of colon. A problem is that questionnaire surveys from various practice settings have suggested that the number of biopsies taken by endoscopists often falls short of recommended guidelines. ${ }^{35,36}$

\section{Microscopic classification of dysplasia}

Gastrointestinal dysplasia is defined microscopically as replacement of the normal intestinal epithelium by a neoplastic, but noninvasive, epithelium. ${ }^{37}$ It is synonymous with the term "intraepithelial neoplasia" used in other organ systems. A standardized histological classification system divides dysplasia in IBD into five categories: negative for dysplasia, indefinite for dysplasia, low-grade dysplasia (LGD), high-grade dysplasia (HGD) or invasive cancer. ${ }^{37} \mathrm{~A}$ further subdivision of the indefinite category into probably negative or probably positive is considered optional by many pathologists and is rarely used. The diagnostic category "indefinite for dysplasia" is an acknowledgment of the difficulty pathologists face sometimes in discriminating between dysplasia and reactive epithelial changes seen in actively inflamed colons, although experienced pathologists can usually discriminate between the two.

The distinction between grades of dysplasia is fairly subjective, resulting in inconsistency among pathologists in the diagnosis of dysplasia on biopsy. The best agreement levels tend to occur at the two extremes of negative and HGD, and the poorest levels in the two gray zones between LGD and HGD and on either side of indefinite for dysplasia. From a practical standpoint, it has been recommended that diagnoses carrying serious management implications be reviewed by at least one additional pathologist with expertise in this area. ${ }^{38}$

\section{BIOLOGY OF DYSPLASIA}

\section{Natural history of dysplasia}

The natural history of dysplasia is a key factor contributing to the outcome and success of surveillance. The model shown in Fig. 1 suggests that colitic mucosa progresses in a systematic fashion: no dysplasia, indefinite dysplasia, LGD, HGD, and finally invasive cancer. While this might be a useful paradigm which facilitates the study of cancer in IBD, it remains unclear whether dysplasia of one grade necessarily "progresses" (or might even "regress") to another grade. For example, there are instances whereby patients undergoing regular colonoscopic surveillance have developed CRC without any prior dysplasia, and, likewise, it is not necessary for LGD to progress to HGD before cancer arises in the colon. ${ }^{39,40}$ This highlights the need to develop markers that are complementary to dysplasia for predicting CRC risk in IBD patients -- a subject of ongoing investigation.

In the meantime, we currently rely upon the histological identification of dysplasia to make management decisions. Refinements in interpreting dysplasia based on the 1983 standardized histological criteria ${ }^{37}$ have enabled a more accurate prediction of which patients are more likely to progress to advanced neoplasia by excluding those whose biopsies only show reactive changes secondary to inflammation. This was amply illustrated by the St. Marks group who found that the 5-year cumulative rate of progression from LGD to HGD or cancer rose from $16 \%$ to $54 \%$ once biopsies were more precisely reclassified. ${ }^{41,42}$

When dysplasia of any grade is detected, there is a chance that CRC is already present in the colon. For example, in a review of ten prospective surveillance trials, $42 \%$ (10 of 24) patients who underwent colectomy because of HGD, and 16\% (3 of 19) patients with LGD who underwent immediate colectomy had synchronous CRC. ${ }^{30}$ Ullman and colleagues at The New York Mount Sinai Hospital performed a retrospective cohort analysis of the outcome of 46 patients with UC who had flat LGD. ${ }^{40}$ They found that $27 \%$ (3 of 11) patients who underwent colectomy within 6 months of the initial detection of flat LGD had a finding of cancer or HGD. More recently, Rutter et al. from the St. Mark's Hospital reported $20 \%$ of patients with LGD who proceeded to colectomy had concurrent adenocarcinoma and 39\% who had follow-up of the LGD progressed to subsequent HGD or CRC. ${ }^{15}$ One possible explanation for why someone with flat LGD might already have CRC is a recently described pathological entity termed low-grade tubuloglandular adenocarcinoma (LGTGA). ${ }^{43}$ This special histological type of adenocarcinoma, which may account for as many as $11 \%$ of all CRCs in IBD, is characterized by malignant glands with low-grade cytological features and an absence or paucity of desmoplastic reaction. The mucosa overlying these tumors and surrounding them 

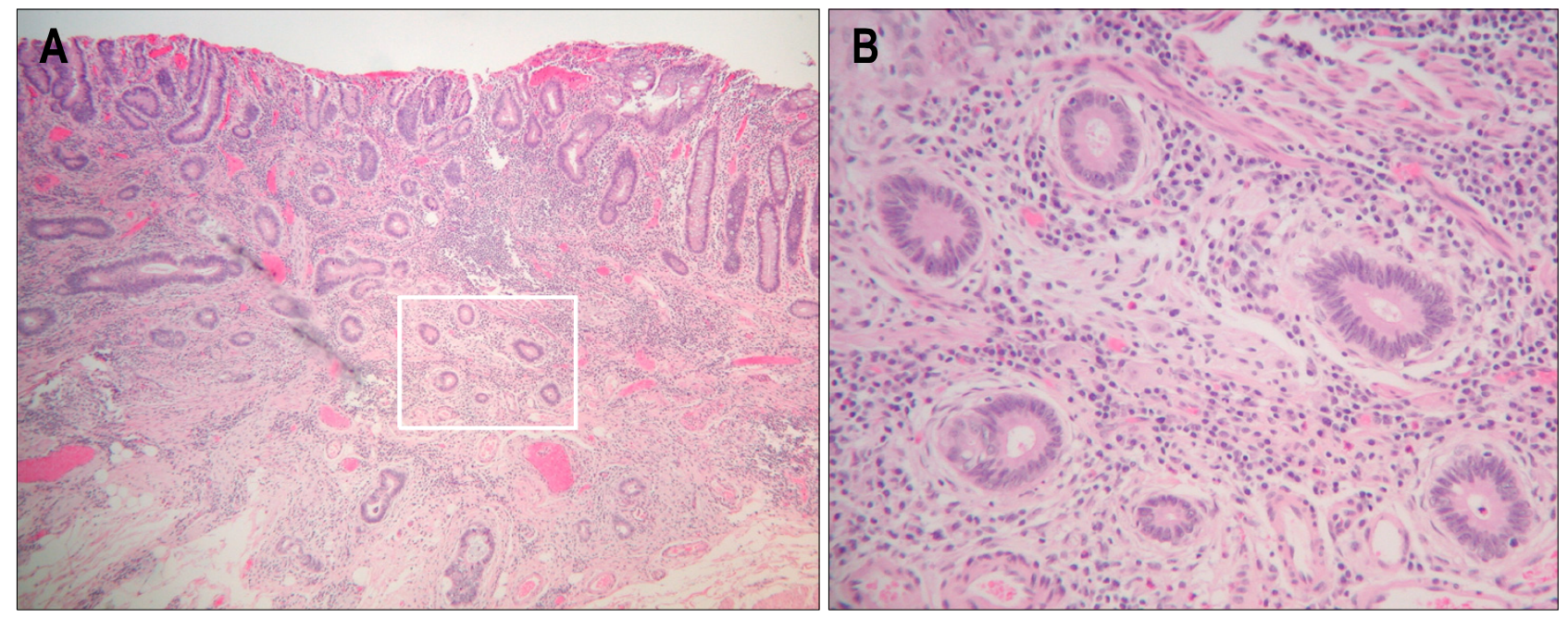

Fig. 2. Invasive low-grade tubuloglandular adenocarcinoma in a patient with ulcerative colitis. (A) The cancer arises directly from mucosa that exhibits low-grade dysplasia on the surface. Biopsies of the surface would be interpreted as flat LGD. (B) High power view of inset in left panel. The invasive, malignant glands are well differentiated and manifest rather low-grade cytological features. There is little or no desmoplastic reaction around the glands. Courtesy of Noam Harpaz, MD, PhD. (and Ref. 43, with permission).

shows LGD or only focal HGD, suggesting that these lesions arise directly from LGD (Fig. 2).

Assuming that early colectomy is not performed, what is the subsequent rate of progression? In patients with HGD, $32 \%$ were found to have CRC after some follow-up period. ${ }^{30}$ Therefore, unless HGD is found in an endoscopically resectable lesion, most physicians recommend colectomy for HGD. For those with LGD, the probability of eventually (time frame not specified) progressing to HGD or CRC was $16-29 \% .^{30}$ Some studies have reported more specifically on the time frame of progression after finding LGD. Data from St. Mark's Hospital indicate that the 5-year cumulative probability of progressing from LGD to HGD or cancer is $54 \% .{ }^{41}$ Strikingly similar results were obtained from The Mount Sinai Hospital, with a 5-year progression rate of 53\% among 46 patients with initial flat LGD. ${ }^{40}$ A recent follow-up analysis from the St. Marks' group indicates slightly lower, but still substantial rates of progression. ${ }^{15}$ Likewise, a series of 18 patients with LGD followed at the Mayo Clinic demonstrated a 33\% 5-year progression rate. ${ }^{44}$ Despite these rather similar results from three different patient populations, some authors have reported substantially lower rates of progression for LGD. Befrits et al. followed 60 patients with flat LGD from the Karolinska Institute in Sweden and found that none developed cancer and only 2 cases of progressed to HGD in DALMs over a mean follow-up period of 10 years. ${ }^{45}$ In the series from Leeds, U.K., Lim et al. reported that only $3 / 29(10 \%)$ patients with LGD progressed to HGD or cancer after 10 years. ${ }^{46}$ It is worth noting that in the lat- ter two studies, the designation of LGD included specimens that were interpreted prior to the 1983 consensus guidelines, so they might have included cases with indefinite dysplasia.

Due to uncertainty in the outcome of flat LGD, it has been difficult to achieve consensus on its proper management. Hence, competing options should be discussed with each patient. A patient confirmed to have multifocal, flat LGD (2 or more biopsies with flat LGD from a single screening or surveillance examination) or repeated, flat LGD ( 2 or more examinations with at least a single focus of flat LGD), should be strongly encouraged to undergo prophylactic total proctocolectomy. Furthermore, even patients with confirmed unifocal LGD (only 1 biopsy positive for flat LGD in a screening or surveillance examination) should be considered for prophylactic proctocolectomy, since evidence indicates that 5-year rate of progression to HGD or CRC in this patient group seems to be similar to that of multifocal LGD. ${ }^{40}$

\section{THE MANAGEMENT OF DYSPLASIA}

\section{Pros and Cons of surveillance}

Once a decision is made to place a patient under surveillance, it is recommended that the patient formally agree to enter such a program and verbalize that he/she is willing to comply. Patients must be made to understand the limitations of surveillance and accept the concept that despite their own cooperation, dysplasia and cancer can still arise even in the hands of skilled endoscopists and pathologists. The best proof that surveillance 
colonoscopy effectively reduces CRC mortality would come from a prospective, randomized, controlled trial in which patients with longstanding IBD would undergo colonoscopic surveillance, whereas controls matched for a similar risk profile would not. However, due to ethical, financial and practical limitations, this type of study will never occur. We must therefore rely on retrospective studies for insights as to the efficacy of surveillance colonoscopy. The best evidence that colonoscopy reduces CRC mortality in UC comes from case-control studies. In one such study, Karlen and colleagues identified 2 of 40 patients with UC and 18 of 102 controls who died because of CRC and had undergone at least one surveillance colonoscopy. CRC mortality was reduced by as much as $78 \%$, although this did not reach statistical significance. ${ }^{47}$ Another study found a similar degree of protection. ${ }^{48}$ In these studies, a protective effect was found for individuals who had even one or two surveillance exams. There is also good evidence from prospective, albeit uncontrolled, studies of surveillance colonoscopy that in general, patients who comply with surveillance have cancers detected at earlier stages compared to those who do not comply. ${ }^{49}$

Even though cancers might still arise within a surveillance program, the gastroenterology community has put its faith in surveillance colonoscopy to prevent CRC in IBD patients. However, surveillance has its limitations. For one thing, there are low rates of observer agreement for the histopathologic interpretation of biopsy specimens between general pathologists and GI pathologists, or even among expert GI pathologists. ${ }^{37,50}$ Endoscopists often fail to take a sufficient number of biopsies to exclude the presence of dysplasia or cancer. ${ }^{36}$ Unlike the dysplastic sporadic adenoma which typically assumes a discrete, polypoid shape surrounded by normal mucosa, dysplasia in the colitic colon can be difficult to discern. This may be particularly troublesome in a colon that is replete with inflammatory pseudopolyps. Furthermore, there is poor understanding of dysplasia amongst trained gastroenterologists. A survey of practicing gastroenterologists and senior GI fellows in the U.S. found that only $19 \%$ of respondents correctly identified dysplasia as neoplastic change. ${ }^{35}$ Patient drop-out or non-compliance with surveillance contributes importantly to CRC mortality in IBD, ${ }^{41,46}$ and this must be considered when embarking upon, or continuing, a course of surveillance in individual patients.

\section{Surveillance strategy}

Despite the limitations of surveillance colonoscopy, dysplasia remains the best marker for managing cancer risk in IBD. A consensus document was recently drafted by an international panel of experts. ${ }^{38}$ After approximately 7-8 years of colitis, patients should undergo an "initial" surveillance colonoscopy to determine the extent of colitis and check for neoplasia. The entire colon should be examined, with approximately 4 biopsies taken every $10 \mathrm{~cm}$. Some experts suggest taking more biopsies in the distal rectosigmoid (e.g. approximately every $5 \mathrm{~cm}$ ) since the distribution of neoplasia in UC still shows a distal predominance. Biopsies should be taken from flat mucosa, but if any raised or suspicious lesions are encountered, these should be removed if possible and processed in separate specimen containers (with additional biopsies taken near the base of the polyp). If a patient is experiencing moderate-severe colitis symptoms, one option is to control the inflammation medically prior to performing the examination in order to minimize difficulties with the histological interpretation of dysplasia. However, one should not defer the colonoscopy too long, since many expert pathologists now feel that they can readily interpret dysplasia even in the presence of active inflammation.

Fig. 3 depicts a recommended surveillance strategy. If no dysplasia is detected, the examination should be repeated in 1-2 years. This interval comes in part from studies reporting that interval cancers can develop within 2 years after a surveillance examination. ${ }^{41}$ If indefinite dysplasia is reported, the nature of the uncertainty should be discussed with the pathologist. If the suspicion of dysplasia is high (i.e., probably positive), repeat biopsy within 3-6 months or less may be indicated; if low, the interval can be lengthened to every 6-12 months. If LGD is detected in a discrete polyp that can be readily resected endoscopically and there is no flat dysplasia immediately adjacent to the polyp or elsewhere in the colon, surveillance can be continued, although the frequency of examinations can be temporarily reduced to every 3-6 months, particularly to re-evaluate the area of polypectomy. Tattoo of the polypectomy site is advised to permit relocalization of the area on subsequent exams. If LGD is detected in flat mucosa (whether unifocal or multifocal), and is confirmed by a second expert GI pathologist, colectomy should be considered. If for some reason colectomy is deferred, repeat surveillance exams should be undertaken within 3-6 months or less. However, the patient should be advised that a negative subsequent examination is no assurance of safety, and that temporizing until there is histological progression to HGD or cancer as the indication for colectomy is risky. A patient in whom flat HGD or adenocarcinoma is found and confirmed by two expert pathologists should undergo colectomy unless serious co-morbidities dictate otherwise. If HGD is diagnosed in an adenoma-like polyp but it is completely removed without evidence of flat dysplasia in the adjacent mucosa 


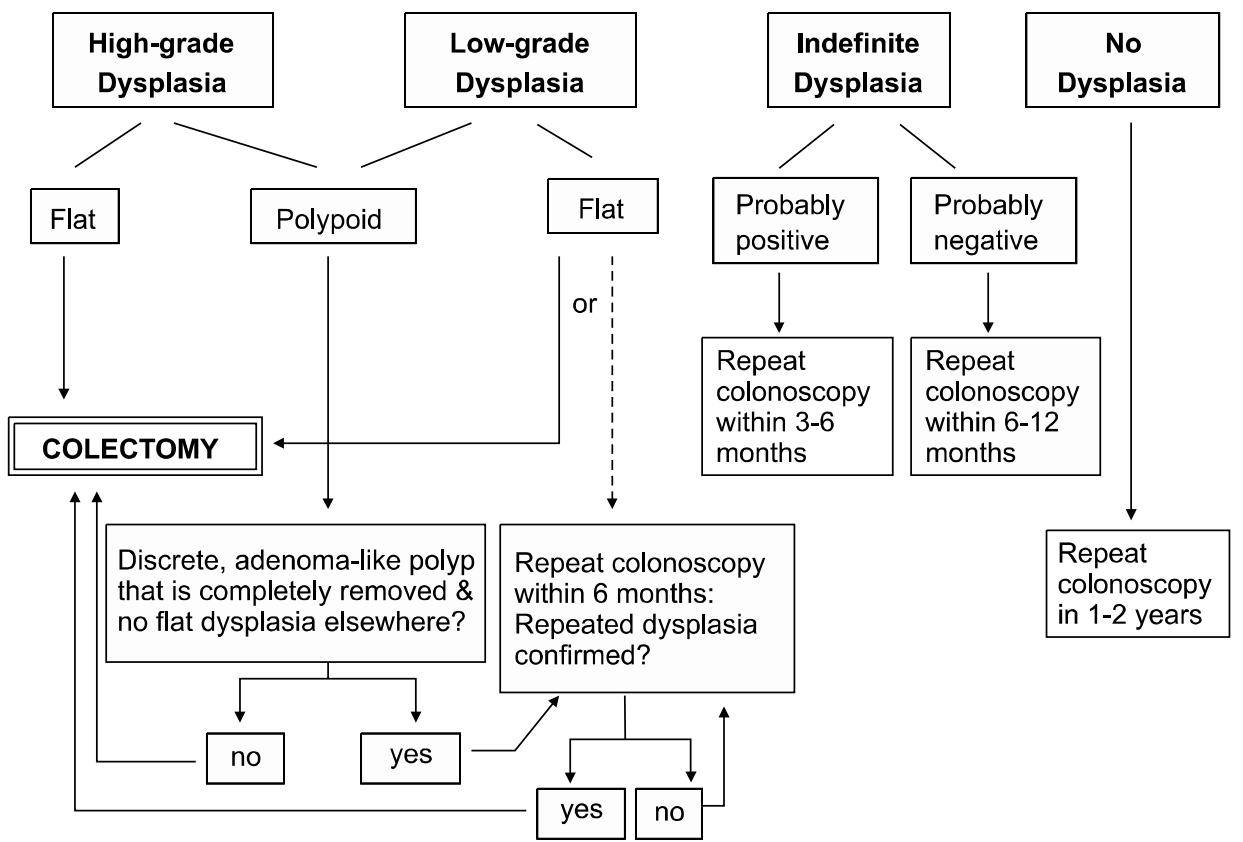

Fig. 3. Suggested management scheme for dysplasia in IBD. Modified from Ref. 61, with permission. or elsewhere in the colon, continued surveillance can be entertained. As with any set of recommendations, decisions should be individualized according to the situation of the patient. Hopefully, strategies for surveillance will become more refined as more knowledge of the natural history of dysplasia is obtained.

Patients who have only had small intestinal Crohn's disease without colonic involvement are not considered to be at high risk for CRC. For patients with Crohn's colitis, much less is known. To date, only one practice-based retrospective surveillance study has been reported in patients with Crohn's colitis. ${ }^{51}$ Of 259 patients with Crohn's colitis affecting at least one-third of the colon for at least 8 years, $16 \%$ were found to have dysplasia or cancer over a 16 year period in which 663 examinations were performed, and there were no cancer deaths. A followup study has found that after a negative screening colonoscopy in Crohn's colitis, the cumulative risk of detecting any dysplasia was $25 \%$ by the tenth surveillance exam, and $7 \%$ for detecting flat HGD or cancer by the ninth surveillance exam. ${ }^{52}$ While we await additional data on the subject, it seems wise to follow a UC-based surveillance strategy for patients with at least 8 years of substantial Crohn's colitis. An important question that remains to be clarified is whether patients with dysplasia or cancer in the setting of segmental Crohn's colitis can undergo segmental resection of the involved area or should proceed to a more extensive UC-like surgical approach. Another dilemma encountered with Crohn's colitis more so than with UC is the management of strictures. Finding a stricture in a colon of a patient with UC usually means an underlying malignancy, especially if the stricture is causing symptoms, and is located in the proximal colon. ${ }^{53}$ However, since most strictures in Crohn's colitis are benign, the patient can often be managed conservatively. Surveillance of such patients often requires using a narrower colonoscope or sometimes dilating a stricture to visualize the proximal mucosa. ${ }^{51}$ Consideration should be given to adding brush cytology of strictures to regular forceps biopsies, and performing a barium enema to evaluate for colonic wall irregularity.

\section{New endoscopic surveillance techniques}

Since flat dysplasia may not be visible during standard endoscopic viewing of the colon mucosa, this has given rise to several innovative techniques which may allow for improved detection of dysplasia at colonoscopy. The best studied technique is chromoendoscopy, which involves spraying dye on the colonic mucosa, often in combination with high resolution or high magnification colonoscopies, to permit a more detailed examination of the mucosa. The first large study to examine chromoendoscopy in the evaluation of dysplasia in IBD was by Kiesslich and colleagues. ${ }^{54}$ In this study, 165 patients with colitis for at least 8 years were randomized either to conventional colonoscopy with biopsies taken every $10 \mathrm{~cm}$ or examination with methylene blue dye spray and high magnification colonoscopy. Forty-six neoplastic lesions including 4 cancers and 10 HGDs were detected. The rate of neoplasia detection was significantly higher in the chromoendoscopy group. Furthermore, of the 32 neoplastic lesions detected in the chromoendoscopy group, while all 

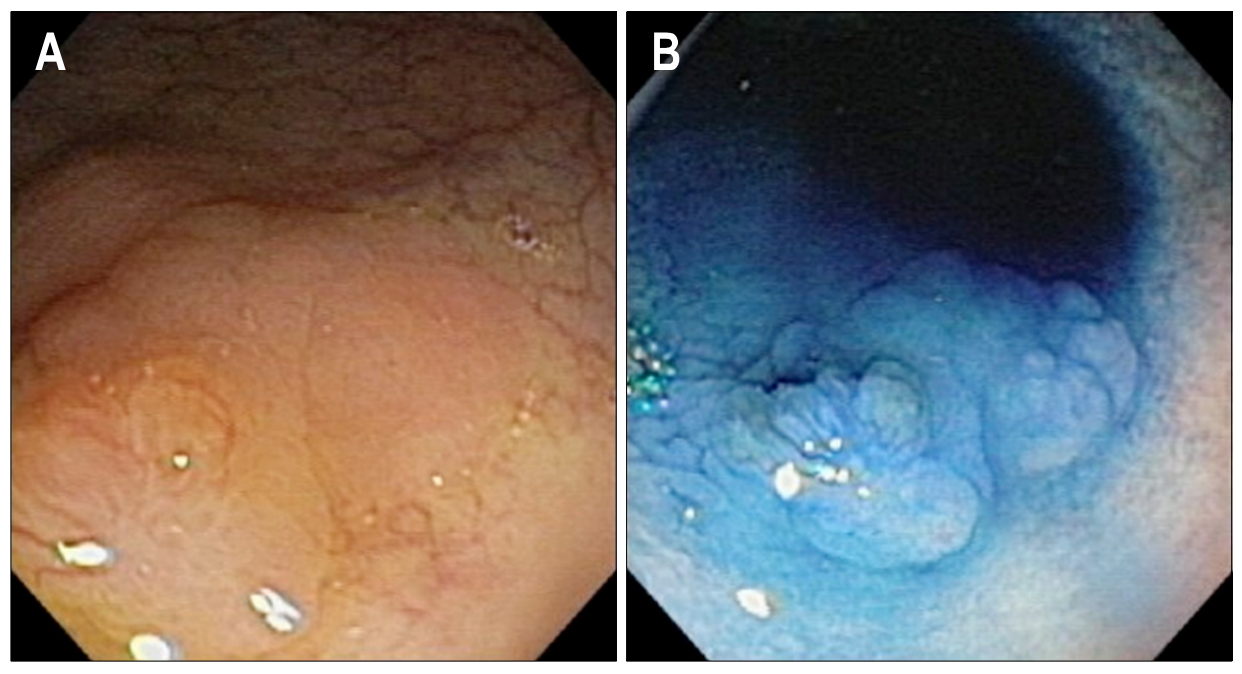

Fig. 4. A slightly raised, but poorly visible flat dysplastic lesion with LGD in a patient with longstanding UC (A). After dye spray with methylene blue, the lesion and its margin are much better defined (B). Courtesy of Jerome D. Waye, M.D.

cancers were detected prior to chromoendoscopy, 21 neoplasms were only identified after the application of the dye. An example of the ability of methylene blue dye spray to enhance visualization of a dysplastic lesion is shown in Fig. 4.

A subsequent study by Rutter and colleagues examined 100 consecutive patients with longstanding colitis. ${ }^{55}$ Each patient served as their own control by undergoing back-to-back colonoscopies, first with a conventional exam then chromoendoscopy with indigo carmine. During the conventional colonoscopy, non-targeted biopsies were taken every $10 \mathrm{~cm}$. No dysplasia was detected in the 2904 non-targeted biopsies. However, 7 dysplastic lesions were detected in the 157 targeted biopsies. While this study showed that chromoendoscopy with indigo carmine improved the detection rate for dysplasia, the specificity of chromoendoscopy for dysplasia in this study was poor.

Hurlstone and colleagues examined 350 patients with longstanding colitis. ${ }^{56}$ Each patient received a standard colonoscopy with 4 quadrant random biopsies every $10 \mathrm{~cm}$ followed by targeted biopsies after application of indigo carmine and evaluation with high magnification colonoscopes. The rates of detection of neoplasia were compared to 350 matched controls that previously underwent conventional colonoscopy. 69 neoplastic lesions were detected in the chromoendoscopy group compared to 24 in the control group (statistically significant). Seventy nine percent of the neoplastic lesions in the chromoendoscopy group were only detected after the application of the dye spray. Twenty dysplastic lesions were detected in the 12850 non-targeted biopsies vs. 49 dysplastic lesions in the 644 targeted biopsies. Thus, this study was similar to the Kiesslich study in showing a higher detection rate of neoplasia in the chromoendoscopy aided group.

Marion and colleagues studied 102 patients with UC and Crohn's colitis first by standard colonoscopy with random four-quadrant biopsies every $10 \mathrm{~cm}$, followed by targeted biopsies of lesions before dye-spray, and then targeted biopsies of lesions detected after dye spray. ${ }^{57}$ Targeted biopsies after dye spray revealed significantly more dysplasia (16 patients with LGD, 1 with HGD) than random biopsies (3 patients with LGD), and more than the targeted, non-dye spray group (8 patients with LGD, 1 with HGD).

Despite these promising results several questions remain about the use of chromoendoscopy in the detection of dysplasia. Although the dye is readily available, the magnification or high resolution colonoscopes used in these studies may not be readily available in many endoscopy units. In addition, the above studies were performed by expert endoscopists with specialized training in chromoendoscopy. It is not clear what degree of training or experience is needed for endoscopists to become proficient in these techniques. Finally, although chromoendoscopy seems to increase the detection of dysplasia, it is still unknown if this will change outcomes in this population of patients. Overall chromoendoscopy holds promise as a technique to improve screening detection of dysplasia over conventional colonoscopy, but many important questions remain unanswered.

Narrow Band Imaging (NBI) is a novel endoscopic technique in which a filter alters the light spectrum increasing the contribution of the shorter wavelength blue light. This allows for detailed imaging of the colonic mucosa similar to resolution obtained with chromoendoscopy but without the need for dye. A pilot study from the Netherlands examined 42 patients with longstanding colitis who underwent back-to-back conventional colonoscopy with random and targeted biopsies of suspicious lesions, plus NBI colonoscopy with targeted biopsies of 
suspicious lesions. ${ }^{58}$ The investigators found that while more suspicious lesions were found with NBI as compared to conventional colonoscopy, the rate of dysplasia detection did not differ between the two methods. Other emerging endoscopic techniques that are being investigated for dysplasia screening are confocal endomicroscopy $^{59}$ and autoflourescence imaging. ${ }^{60}$

\section{Chemoprevention}

Despite the relative protection afforded by surveillance colonoscopies in IBD, there are still patients who develop CRC despite seemingly optimal surveillance. This raises the issue of whether chemoprevention in the form of either medications or dietary supplements might help reduce the risk of CRC in IBD. ${ }^{61}$

Aspirin and other NSAIDs markedly reduce the incidence and mortality from, sporadic CRC. Since many patients with IBD take NSAIDs in the form of 5-aminosalicylates (5-ASA), it is reasonable to ask whether 5-ASA compounds might also be protective. Although no study to date has been performed in a prospective manner specifically to address this question, the available data suggest that this may be so. ${ }^{62}$ If 5-ASA compounds prevent colonic neoplasia by suppressing inflammation, it follows that other anti-inflammatory medications used in IBD patients should also be protective against CRC. Although one study reported that the use of systemic steroids, and even topical steroids resulted in a significant CRC risk reduction $^{48}$ and others confirm this observation, ${ }^{63}$ steroids cannot be used long-term for chemoprevention due to safety issues. The immunomodulators 6-mercaptopurine and azathioprine appear to have no chemopreventive activity. $^{64}$

In the setting of sporadic CRC, low folate intake has been associated with an increased risk for developing colorectal adenomas and carcinomas. ${ }^{65,66}$ Patients with chronic IBD are predisposed toward folate deficiency because of inadequate nutritional intake, excessive intestinal losses with active disease, and reduced intestinal absorption from competitive inhibition from sulfasalazine use. Results of two studies suggest a trend toward protection against CRC in folate users, although neither study demonstrated statistical significance. ${ }^{67,68}$ Nonetheless, since it is rather safe and inexpensive, folate supplementation should be considered for CRC risk reduction in patients with longstanding IBD.

In animal models of colon carcinogenesis, ursodiol inhibits carcinogenesis - an effect that may be due to the ursodiol reducing the colonic concentration of the secondary bile acid deoxycholic acid. Ursodiol also has anti-oxidant activity. A study of UC patients with PSC demon- strated that ursodiol use was strongly associated with decreased prevalence of colonic dysplasia. ${ }^{69}$ This protective effect remained after adjusting for duration of colitis, age at onset of colitis, and sulfasalazine use. In a follow-up to the randomized, placebo-controlled trial at the Mayo Clinic, 52 patients with chronic PSC and chronic UC (mean 13 years) were followed for a total of 335 person-years. ${ }^{70}$ Ursodiol use was associated with a significant protection against the development of dysplasia and cancer $(R R=0.26, p=0.034)$. Currently, however, we do not know whether ursodiol can prevent neoplastic progression in UC patients without PSC.

Recently, there has been interest in the role statins may play as chemopreventive agents in a variety of cancers. In a population-based case-control study of patients who had diagnosis of CRC in northern Israel between 1998-2004, statin therapy was associated with a modest reduction in CRC in the non-IBD population, but a substantial $94 \%$ risk reduction in patients with IBD was observed in a subset analysis of a small number of patients. ${ }^{71}$ Further studies are needed to verify this benefit.

\section{Cancer after colectomy}

Patients with UC who have undergone total proctocolectomy with ileal pouch anal-anastomosis (IPAA) have a very small risk of dysplasia arising within the ileal mucosa of the pouch itself. The risk is thought to be higher in patients with chronic pouchitis and associated severe villous atrophy, ${ }^{72}$ but this has not been shown in all series. ${ }^{73}$ Indeed, a study of 160 patients who underwent biopsy a total of 222 times with an average surveillance time of 8.4 years after surgery showed that in 1800 pouch-years of surveillance, only one patient had focal LGD of the pouch. ${ }^{74}$ The risk of neoplasia is greater in the anal transitional mucosa between the pouch and the anal canal, particularly if a cuff of rectal mucosa has been left, and if the indication for the IPAA was rectal dysplasia or cancer. ${ }^{75}$ While there are currently no guidelines for endoscopic surveillance after an IPAA procedure, in those patients who have chronic pouchitis and severe villous atrophy or whose original indication for IPAA was dysplasia or cancer, a program of periodic endoscopy with biopsies, paying particular attention to any anal transition zone, is reasonable.

\section{FUTURE DIRECTIONS}

The future looks promising with respect to new developments in the management of CRC risk in IBD. Chromoendoscopy is likely to be used more for manage- 
ment, but whether the predictable increase in the yield of dysplasia will alter the overall natural history remains to be studied. In the modern era of molecular diagnostics, tissue and even stool samples of patients with IBD can be investigated for molecular alterations. For example, because there is often widespread genomic instability throughout the colon of IBD patients, it may be possible to analyze rectal biopsies by DNA fingerprinting or fluorescence in-situ hybridization methods ${ }^{76}$ to identify patients at particularly high risk. The advent of technology to extract human DNA from stool and look for specific DNA mutations associated with sporadic colon carcinogenesis $^{77}$ implies that a similar approach may be worthwhile in IBD patients. It is anticipated that refinements in our knowledge of cancer biology, clinical practice, and molecular discovery will bring a new level of sophistication to the management of patients with longstanding IBD and lower the incidence of CRC in this high-risk population.

\section{REFERENCES}

1. Crohn BB, Rosenberg $\mathrm{H}$. The sigmoidoscopic picture of chronic ulcerative colitis. Am J Med Sci 1925;170:220-228.

2. Devroede GJ, Taylor WF, Sauer WG, et al. Cancer risk and life expectancy of children with ulcerative colitis. N Engl J Med 1971;285:17-21.

3. Winther $\mathrm{KV}$, Jess $\mathrm{T}$, Langholz $\mathrm{E}$, et al. Survival and cause specific mortality in ulcerative colitis: follow-up of a population based cohort in Copenhagen County. Gastroenterology 2003;125:1576-1582.

4. Eaden JA, Abrams KF, Mayberry JA. The risk of colorectal cancer in ulcerative colitis; a meta-analysis. Gut 2001;48: 526-535.

5. Palli D, Trallori G, Bagnoli S, et al. Hodgkins Disease risk is increased in patients with ulcerative colitis. Gastroenterology 2000;119:647-653.

6. Bernstein $\mathrm{CN}$, Blanchard JF, Kliewer E, et al. Cancer risk in patients with inflammatory bowel disease; a population based study. Cancer 2001;91:854-862.

7. Latkos L, Mester M, Erdelyi Z, et al. Risk factors for ulcerative colitis associated colorectal cancers in a Hungarian cohort of patients with ulcerative colitis: results of a population based study. Inflamm Bowel Dis 2006;12:205-211.

8. Loftus EV. Epidemiology and risk factors for colorectal dysplasia and cancer in ulcerative colitis. Gastroenterol Clin North Am 2006;35:517-531.

9. Ekbom A, Helmick C, Zack M, et al. Ulcerative colitis and colorectal cancer: a population based study. N Engl J Med 1990;323:1228-1233.

10. Karlen P, Lofberg R, Brostrom O, et al. Increased risk of cancer in ulcerative colitis: a population based cohort study. Am J Gastroenterol 1999;94:1047-1052.

11. Winther KV, Jess T, Langholz E, et al. Long term risk of cancer in ulcerative colitis: a population based cohort study from Copenhagen county. Clin Gastroenterol Hepatol 2004;2:1088-1095.
12. Jess T, Loftus EV, Velayos FS, et al. Risk of intestinal cancer in inflammatory bowel disease: a population based study from Olmsted County, Minnesota. Gastroenterology 2006;130:1039-1046.

13. Lindberg J, Stenling R, Palmqvist R, et al. Efficiency of colorectal cancer surveillance in patients with ulcerative colitis; 26 years of experience in a patient cohort from a defined population area. Scand J Gastroenterol 2005;40: 1076-1080.

14. Jess T, Loftus EV, Velayos FS, et al. Incidence and prognosis of colorectal dysplasia in inflammatory bowel disease: a population based study from Olmsted County, Minnesota. Inflamm Bowel Dis 2006;12:669-676.

15. Rutter MD, Saunders BP, Wilkinson KH, et al. Thirty year analysis of a colonoscopic surveillance program for neoplasia in ulcerative colitis. Gastroenterology 2006;130: 1030-1038.

16. Bernstein CN, Blanchard JF, Kliewer E, Wajda A. Cancer risk in patients with inflammatory bowel disease: a population-based study. Cancer 2001;91:854-862.

17. Choi PM, Zelig MP. Similarity of colorectal cancer in Crohn's disease and ulcerative colitis: implications for carcinogenesis and prevention. Gut 1994;35:950-954.

18. Ekbom A, Helmick C, Zack M, Adami HO. Ulcerative colitis and colorectal cancer: a population-based study. N Engl J Med 1990;323:1228-1233.

19. Gyde SN, Prior P, Allan RN, et al. Colorectal cancer in ulcerative colitis: a cohort study of primary referrals from three centres. Gut 1988;29:206-217.

20. Heuschen UA, Hinz U, Allemayer EH, et al. Backwash ileitis is strongly associated with colorectal carcinoma in ulcerative colitis. Gastroenterology 2001;120:841-847.

21. Haskell H, Andrews CW, Reddy SI, et al. Pathological features and clinical significance of backwash ileitis in ulcerative colitis. Am J Surg Pathol 2005;29:1472-1481.

22. Rutter M, Saunders B, Wilkinson K, et al. Severity of inflammation is a risk factor for colorectal neoplasia in ulcerative colitis. Gastroenterology 2004;126:451-459.

23. Gupta RB, Harpaz N, Itzkowitz S, et al. Histologic inflammation is a risk factor for progression to colorectal neoplasia in ulcerative colitis: a cohort study. Gastroenterology 2007;133:1099-1105.

24. Mathy C, Schneider K, Chen YY, et al. Gross versus microscopic pancolitis and the occurrence of neoplasia in ulcerative colitis. Inflamm Bowel Dis 2003;9:351-355.

25. Kornfeld D, Ekbom A, and Ihre T. Is there an excess risk for colorectal cancer in patients with ulcerative colitis and concomitant primary sclerosing cholangitis? A population based study. Gut 1997;41:522-525.

26. Shetty K, Rybicki L, Brzezinski A, Carey WD, Lashner BA. The risk for cancer or dysplasia in ulcerative colitis patients with primary sclerosing cholangitis. Am J Gastroenterol 1999;94:1643-1649.

27. Lundqvist K, Broome U. Differences in colonic disease activity in patients with ulcerative colitis with and without primary sclerosing cholangitis: a case control study. Dis Colon Rectum 1997;40:451-461.

28. Pardi DS, Loftus EV, Kremers WK, et al. Ursodeoxycholic acid as a chemopreventative agent in patients with ulcerative colitis and primary sclerosing cholangitis. Gastroenterology 2003;124:889-893. 
29. Blackstone MO, Riddell RH, Rogers BHG, Levin B. Dysplasia-associated lesion or mass (DALM) detected by colonoscopy in long-standing ulcerative colitis: an indication for colectomy. Gastroenterology 1981;80:366-374.

30. Bernstein CN, Shanahan F, Weinstein WM. Are we telling patients the truth about surveillance colonoscopy in ulcerative colitis? Lancet 1994;343:71-74.

31. Rubin PH, Friedman S, Harpaz N, et al. Colonoscopic polypectomy in chronic colitis: conservative management after endoscopic resection of dysplastic polyps. Gastroenterology 1999;117:1295-1300.

32. Engelsgjerd M, Farraye FA, Odze RD. Polypectomy may be adequate treatment for adenoma-like dysplastic lesions in chronic ulcerative colitis. Gastroenterology 1999;117:12881294.

33. Odze RD, Farraye FA, Hecht JL, Hornick JL. Long-term follow-up after polypectomy treatment for adenoma-like dysplastic lesions in ulcerative colitis. Clin Gastroenterol Hepatol 2004;2:534-541.

34. Rubin CE, Haggitt RC, Burmer GC, et al. DNA aneuploidy in colonic biopsies predicts future development of dysplasia in ulcerative colitis. Gastroenterology 1992;103:16111620.

35. Bernstein CN, Weinstein WM, Levine DS, Shanahan F. Physicians' perceptions of dysplasia and approaches to surveillance colonoscopy in ulcerative colitis. Am J Gastroenterol 1995;90:2106-2114.

36. Eaden JA, Ward BA, Mayberry JF. How gastroenterologists screen for colonic cancer in ulcerative colitis: an analysis of perfomance. Gastrointest Endosc 2000;51:123-128.

37. Riddell RH, Goldman H, Ransohoff DF, et al. Dysplasia in inflammatory bowel disease: standardized classification with provisional clinical applications. Hum Pathol 1983;14: 931-968.

38. Itzkowitz SH, Present DH, Crohn's and Colitis Foundation of America Colon Cancer in IBD Study Group. Consensus conference: Colorectal cancer screening and surveillance in inflammatory bowel disease. Inflamm Bowel Dis 2005;11: 314-321.

39. Harpaz N, Talbot IC. Colorectal cancer in idiopathic inflammatory bowel disease. Semin Diagn Pathol 1996;13: 339-357.

40. Ullman TA, Croog T, Harpaz N, Sachar D, Itzkowitz S. Progression of flat low-grade dysplasia to advanced neoplasia in patients with ulcerative colitis. Gastroenterology 2003;125:1311-1319.

41. Connell WR, Lennard-Jones JE, Williams CB, et al. Factors affecting the outcome of endoscopic surveillance for cancer in ulcerative colitis. Gastroenterology 1994;107:934-944.

42. Lennard-Jones JE, Melville DM, Morson BC, Ritchie JK, Williams CB. Precancer and cancer in extensive ulcerative colitis: findings among 401 patients over 22 years. Gut 1990;31:800-806.

43. Levi GS, Harpaz N. Intestinal low-grade tubuloglandular adenocarcinoma in inflammatory bowel disease. Am J Surg Pathol 2006;30:1022-1029.

44. Ullman TA, Loftus EV Jr, Kakar S, et al. The fate of low grade dysplasia in ulcerative colitis. Am J Gastroenterol 2002;97:922-927.

45. Befrits R, Ljung T, Jaramillo E, Rubio C. Low-grade dysplasia in extensive, long-standing inflammatory bowel disease; a follow-up study. Dis Colon Rectum 2002;45:615-620.

46. Lim CH, Dixon MF, Vail A, et al. Ten year follow up of ulcerative colitis patients with and without low grade dysplasia. Gut 2003;52:1127-1132.

47. Karlen P, Kornfeld D, Brostrom O, et al. Is colonoscopic surveillance reducing colorectal cancer mortality in ulcerative colitis? A population based case control study. Gut 1998;42:711-714.

48. Eaden J, Abrams K, Ekbom A, Jackson E, Mayberry J. Colorectal cancer prevention in ulcerative colitis: a casecontrol study. Aliment Pharmacol Ther 2000;14:145-153.

49. Griffiths AM, Sherman PM. Colonoscopic surveillance for cancer in ulcerative colitis: a critical review. J Pediatr Gastroenterol Nutr 1997;24:202-210.

50. Melville DM, Jass JR, Morson BC, et al. Observer study of the grading of dysplasia in ulcerative colitis: comparison with clinical outcome. Hum Pathol 1989;20:1008-1014.

51. Friedman S, Rubin PH, Bodian C, et al. Screening and surveillance colonoscopy in chronic Crohn's colitis. Gastroenterology 2001;120:820-826.

52. Friedman S, Rubin PH, Bodian C, Harpaz N, Present DH. Screening and surveillance colonoscopy in chronic Crohn's colitis: results of a surveillance program spanning 25 years. Clin Gastroenterol Hepatol 2008; in press.

53. Gumaste V, Sachar DB, Greenstein AJ. Benign and malignant colorectal strictures in ulcerative colitis. Gut 1992; 33:938-941.

54. Kiesslich R, Frisch J, Holtman M, et al. Methylene blue aided chromoendoscopy for the detection of intraepithelial neoplasia and colon cancer in ulcerative colitis. Gastroenterology 2003;124:880-888.

55. Rutter MD, Saunders BP, Schofield G, et al. Pancolonic indigo carmine dye spraying for the detection of dysplasia in ulcerative colitis. Gut 2004;53:256-260.

56. Hurlstone DP, Sanders DS, Lobo AJ, et al. Indigo carmine assisted high magnification chromoscopic colonoscopy for the detection and characterization of intraepithelial neoplasia and colon cancer in ulcerative colitis: a prospective evaluation. Endoscopy 2005;37:1186-1192.

57. Marion JF, Waye JD, Present DH, et al. Chromoendoscopy-targeted biopsies are superior to standard colonoscopic surveillance for detecting dysplasia in inflammatory bowel disease patients: a prospective endoscopic trial. Am J Gastroenterol 2008; in press.

58. Dekker E, Van den Broek FJC, Reitsma JB, et al. Narrow band imaging compared with conventional colonoscopy for the detection of dysplasia in patients with longstanding ulcerative colitis. Endoscopy 2007;39:216-221.

59. Kiesslich R, Goetz M, Lammersdorf K, et al. Chromoscopy guided endomicroscopy increases diagnostic yield of intraepithelial neoplasia in ulcerative colitis. Gastroenterology 2007;132:874-882.

60. Matsumoto T, Moriyama T, Yao T, et al. Autoflourescence colonoscopy for the diagnosis of dysplasia in ulcerative colitis. Inflamm Bowel Dis 2007;13:640-641.

61. Itzkowitz SH, Harpaz N. Diagnosis and management of dysplasia in patients with inflammatory bowel diseases. Gastroenterology 2004;126:1634-1648.

62. Rubin DT, Cruz-Correa MR, Gasche C, et al. Colorectal cancer prevention in inflammatory bowel disease and the role of 5-aminosalicylic acid: a clinical review and update. 
Inflamm Bowel Dis 2008;14:265-274.

63. Velayos FS, Loftus EV Jr, Jess T, et al. Predictive and protective factors associated with colorectal cancer in ulcerative colitis: A case-control study. Gastroenterology 2006; 130:1941-1949.

64. Matula S, Croog V, Itzkowitz S, et al. Chemoprevention of colorectal neoplasia in ulcerative colitis: the effect of 6-mercaptopurine. Clin Gastroenterol Hepatol 2005;3:10151021.

65. Freudenheim J, Graham S, Marshall J, et al. Folate intake and carcinogenesis of the colon and rectum. Int $\mathrm{J}$ Epidemiol 1991;20:368-374.

66. Giovannucci E, Stampfer MJ, Colditz GA, et al. Folate, methionine, and alcohol intake and risk of colorectal adenoma. J Natl Cancer Inst 1993;85:875-884.

67. Lashner BA, Heidenreich PA, Su GL, Kane SV, Hanauer SB. Effect of folate supplementation on the incidence of dysplasia and cancer in chronic ulcerative colitis. Gastroenterology 1989;97:255-259.

68. Lashner BA, Provencher KS, Seidner DL, Knesebeck A, Brzezinski A. The effect of folic acid supplementation on the risk for cancer or dysplasia in ulcerative colitis. Gastroenterology 1997;112:29-32.

69. Tung BY, Emond MJ, Haggitt RC, et al. Ursodiol use is associated with lower prevalence of colonic neoplasia in patients with ulcerative colitis and primary sclerosing cholangitis. Ann Intern Med 2001;134:89-95.
70. Pardi DS, Loftus EV Jr, Kremers WK, Keach J, Lindor KD. Ursodeoxycholic acid as a chemopreventive agent in patients with ulcerative colitis and primary sclerosing cholangitis. Gastroenterology 2003;124:889-893.

71. Poynter JN, Gruber SB, Higgins PD, et al. Statins and the risk of colorectal cancer. N Engl J Med 2005;352: 2184-2192.

72. Gullberg K, Stahlberg D, Liljeqvist L, et al. Neoplastic transformation of the pelvic pouch mucosa in patients with ulcerative colitis. Gastroenterology 1997;112:1487-1492.

73. Thompson-Fawcett MW, Marcus V, Redston M, Cohen Z, McLeod RS. Risk of dysplasia in Long-term ileal pouches and pouches with chronic pouchitis. Gastroenterology 2001;121:275-281.

74. Herline AJ, Meisinger LL, Rusin LC, et al. Is routine pouch surveillance for dysplasia indicated for ileoanal pouches? Dis Colon Rectum 2003;46:156-159.

75. O'Riordain MG, Fazio VW, Lavery IC, et al. Incidence and natural history of dysplasia of the anal transitional zone after ileal pouch-anal anastomosis: results of a five-year to ten-year follow-up. Dis Colon Rectum 2000;43:1660-1665.

76. Brentnall TA. Molecular underpinnings of cancer in ulcerative colitis. Curr Opin Gastroenterol 2003;19:64-68.

77. Itzkowitz SH, Jandorf L, Brand R, et al. Improved fecal DNA test for colorectal cancer screening. Clin Gastroenterol Hepatol 2007;5:111-117. 